\title{
Exuberance meets harsh realities in the bioprosthetic tissue valve era
}

\author{
Gabriel S. Aldea, MD, ${ }^{\mathrm{a}}$ and Danny Dvir, $\mathrm{MD}^{\mathrm{b}}$
}

\author{
From the Divisions of ${ }^{\mathrm{a} C a r d i o t h o r a c i c ~ S u r g e r y ~ a n d ~}{ }^{\mathrm{b}}$ Cardiology, University of Washington, Seattle, Wash. \\ Disclosures: Authors have nothing to disclose with regard to commercial support. \\ Received for publication Jan 10, 2018; accepted for publication Jan 12, 2018; available ahead of print Feb 23, \\ 2018. \\ Address for reprints: Gabriel S. Aldea, MD, Division of Cardiothoracic Surgery, University of Washington, 1959 \\ NE Pacific St, Box 356310, Seattle, WA 98195-6310 (E-mail: aldea@u.washington.edu) \\ J Thorac Cardiovasc Surg 2018;155:e145-6 \\ $0022-5223 / \$ 36.00$ \\ Copyright (C) 2018 by The American Association for Thoracic Surgery \\ https://doi.org/10.1016/j.jtcvs.2018.01.027
}

This report by Ochiai and colleagues ${ }^{1}$ highlights the consequences of (over) exuberant use of bioprosthetic valves in younger more complex patient populations clashing with the reality of more rapid prosthetic valve degeneration in these same patients. ${ }^{2}$ This paradox and concerning trend portend a tsunami of future long-term challenges. The case demonstrates the inconsistent way we collectively follow up and underreport bioprosthetic valve status with late clinical presentations (mean gradient of $>100 \mathrm{~mm} \mathrm{Hg}$ and aortic valve area $<0.4 \mathrm{~cm}^{2}$ ) and possible negative consequences on outcomes. It finally highlights the challenges, intricacies, and limitations of treating degenerated (open surgical and increasingly transcatheter) valves with transcatheter aortic valve replacement (TAVR) valve-in-valve (ViV) and even more so with valve-in-valve-in-valve ( ViViV).

In this case, the inner diameter of the second surgical aortic valve replacement (SAVR) 23 CE Magna (Edwards Lifesciences, Irvine, Calif) implanted was small (20$21 \mathrm{~mm}$ ). A supra-annular valve may have been associated with lower gradients. ${ }^{3}$ Pin-wheeling, crowding, increased stress, blood residence time, and incomplete opening of a perhaps oversized $\mathrm{ViV}$ all may have contributed to this patient's very early (3 years from implantation) prosthetic valve degeneration with typical fibrosis, pannus, and asymmetric calcification. Placing a second 20-mm Sapien 3 (Edwards Lifesciences) ViViV, although technically easier than SAVR, inevitably resulted in high residual gradients and PPM. Despite presumed (frequently overestimated) risk of a third sternotomy because of the experience of this team, the patient did well with a third SAVR with a 21-mm Magna Ease valve, but again was left with a small prosthetic valve inner diameter (19 $\mathrm{mm}$ ) that will limit future ViV options.

Our own experience mirrors national trends with greater than $90 \%$ use of biologic prostheses ${ }^{4}$ (as in this patient with her first SAVR bioprosthetic valve implanted at the age of 45 years and a second at the age of 59 years). This is commonly driven by an overwhelming patient preference to avoid lifelong warfarin anticoagulation. However, this choice assumes that future technologic evolutions will effectively treat prosthetic valve degeneration and assumes

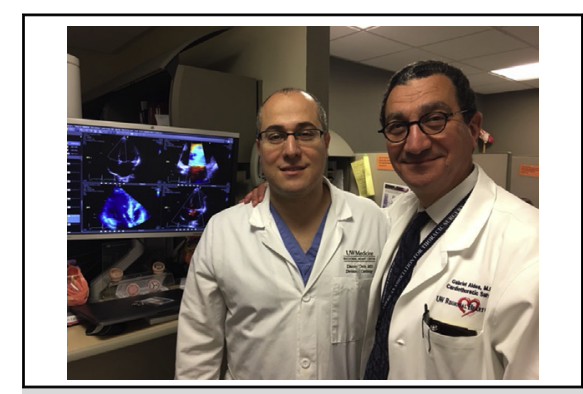

Use of bioprosthetic valves in younger patients will lead to formidable future challenges.

\section{Central Message}

The (over) exuberant use of bioprosthetic valves in younger more complex patient populations portends a tsunami of future long-term challenges.

See Article page e141.

that such therapies will be performed with minimal hemodynamic consequence and periprocedural risk. Such goals remain aspirational. Decisions to proceed with bioprosthetic SAVR are rarely formally reviewed by a multidisciplinary heart team to objectively assess individual anatomy, better inform, and direct initial valve implantation with the goal of reducing risk and simplifying inevitable future reinterventions. Patients with small annuli are particularly at risk for prosthetic-patient mismatch (PPM). These patients have a higher mortality and less clinical improvement after initial valve replacement. In addition, their valves are prone to early degeneration, and performing $\mathrm{ViV}$ in a device with severe PPM is associated with poor clinical outcomes. ${ }^{5,6}$ PPM is a potentially preventable adverse event that could be avoided with annular enlargement procedures or alternatively use of novel prostheses such as sutureless (Perceval; Sorin Livanova; Houston, Tex) and rapid deployment (Intuity, Edwards Lifesciences) valves. Specific patient anatomy (annulus, calcification, coronary artery height, sinuses) and prior surgical valve type (configuration of leaflets, true inner diameters, leaflet height) affect effectiveness and risk of ViV therapy. Newer valves (eg, INSPIRIS, Edwards Lifesciences) have unique design features to facilitate future transcatheter $\mathrm{ViV}$ procedures and can be considered in younger patients who are at high risk for early valve degeneration. 
We believe that current trends for bioprosthetic SAVR and TAVR in younger patients require a more formal reassessment and multipronged approach:

1. Choice of initial bioprosthetic valve and patient specific anatomy need to be formally considered in younger patients (age $<75$ years). In small annuli, this should include consideration for the possible need for root enlargement and the use of novel sutureless and rapid deployment valves.

2. Routine serial echocardiographic follow-up with objective criteria and staging of structural valve degeneration is needed. ${ }^{7}$ This may lead to earlier more effective, less risky reintervention.

3. Redo AVR with mechanical or novel biological prosthesis should be considered in younger patients.

4. ViV (for both surgically implanted and TAVR degenerated valves) requires advanced analytic multidisciplinary interventional imaging and planning to select the ideal durable therapy for an individual patient. Advanced techniques include supra-annular placement of transcatheter $\mathrm{ViV}$, as well as novel approaches currently in investigational device exemption trials such as planned leaflet lacerations to minimize the risk of coronary artery occlusion (BASILICA [Bioprosthetic or Native Aortic Scallop Intentional Laceration to Prevent Iatrogenic Coronary Artery Obstruction] procedure $)^{8}$ and planned ring rupture ${ }^{9}$ to minimize the risk of coronary artery occlusion and PPM in patients who are high risk or poor candidates for repeat SAVR.
Referring and treating physicians and younger patients with implanted tissue valves should understand that their clinical course mirrors that of other chronic diseases and malignancies with requisite lifelong surveillance, repeated stages of recurrence, reinterventions, and remission.

\section{References}

1. Ochiai T, Yoon S-H, Sharma R, Chakravarty T, Nakamura M, Cheng W, et al. Recurrent severe aortic stenosis after transfemoral transcatheter valve-in-valve-in-valve replacement. J Thorac Cardiovasc Surg. 2018; 155:e141-4

2. Goldstone AB, Chiu P, Baiocchi M, Lingala B, Patrick WL, Fischbein MP, et al. Mechanical or biologic prostheses for aortic-valve and mitral-valve replacement. N Engl J Med. 2017;377:1847-57.

3. Azadani AN, Reardon M, Simonato M, Aldea G, Nickenig G, Kornowski R, et al. Effect of transcatheter aortic valve size and position on valve-in-valve hemodynamics: an in-vitro study. J Thorac Cardiovasc Surg. 2017;153:1303-15.

4. Kaneko J, Aranki S. No rat poison for me. J Thorac Cardiovasc Surg. 2017;154: 1542-4.

5. Dvir D, Webb J, Bleiziffer S, Pasic M, Waksman R, Kodali S, et al. Transcatheter aortic valve implantation in failed bioprosthetic surgical valves. JAMA. 2014;312: 162-70.

6. Pibarot P, Simonato M, Barbanti M, Linke A, Kornowski R, Rudolph T, et al. Impact of pre-existent prosthesis-patient mismatch on survival following aortic valve-in-valve procedures. JACC Cardiovasc Interv. 2018; 22:133-41.

7. Dvir D, Bourguignon T, Otto C, Hahn RT, Rosenhek R, Webb JG, et al. Standardized definition of structural valve degeneration for surgical and transcatheter bioprosthetic aortic valves. Circulation. 2018;137:388-99.

8. Khan J, Dvir D, Greenbaum A, et al. Transcatheter laceration of aortic leaflets to prevent coronary obstruction during transcatheter aortic valve implantation: concept to first-in-human. JACC Cardiovasc Interv. 2018 [In press].

9. Chhatriwalla AK, Allen KB, Saxon JT, Cohen DJ, Aggarwal S, Hart AJ, et al. Bioprosthetic valve fracture improves the hemodynamic results of valve-in-valve transcatheter aortic valve replacement. Circ Cardiovasc Interv. 2017;10: https://doi.org/10.1161/CIRCINTERVENTIONS. 